\title{
Spin-current-induced electric field
}

\author{
Qing-feng Sun, ${ }^{1,2}$ Hong Guo, ${ }^{2,1}$ and Jian Wang ${ }^{3}$ \\ ${ }^{1}$ Institute of Physics, Chinese Academy of Sciences, Beijing 100080, China \\ ${ }^{2}$ Center for the Physics of Materials and Department of Physics, McGill University, Montreal, Quebec, Canada H3A 2T8 \\ ${ }^{3}$ Department of Physics, The University of Hong Kong, Pokfulam Road, Hong Kong, China
}

(Received 29 September 2003; revised manuscript received 13 November 2003; published 13 February 2004)

\begin{abstract}
We theoretically investigate properties of the induced electric field of a steady-state spin-current without charge current, using an "equivalent magnetic charge" method. Several general formula for the induced electric field are derived which play the role of "Biot-Savart law" and "Ampere's law." Conversely, a moving spin is affected by an external electric field and we derive an expression for the interaction torque.
\end{abstract}

DOI: 10.1103/PhysRevB.69.054409

PACS number(s): 72.25.-b, 03.50.De

In a traditional electric circuit the number of spin-up and spin-down electrons are the same, and both kinds of electrons move in the same direction under an external electric field. The total spin-current $I_{s}=\sigma\left(I_{\uparrow}-I_{\downarrow}\right)$ is therefore zero, and only the charge current $I_{e c}=e\left(I_{\uparrow}+I_{\downarrow}\right)$ is relevant. When a system includes ferromagnetic materials, or under an external magnetic field, electron spins can be polarized so that the total spin of the system is nonzero. Then, the corresponding charge current is polarized, i.e., current due to spin-up electrons, $I_{\uparrow}$, is not equal to the spin-down current $I_{\downarrow}$, although both kinds of electrons move in the same direction, as schematically shown in Fig. 1(b). This gives a nonzero total spincurrent. Spin-polarized charge-current has been the subject of extensive investigations for last two decades. ${ }^{1,2}$ Recently, a very interesting extreme case of a finite spin-current without charge-current has been investigated by several groups. ${ }^{3-5}$ Such a situation comes about when spin-up electrons move to one direction while an equal number of spindown electrons move to the opposite direction, as schematically shown in Fig. 1(a). Then the total charge-current is identically zero and only a net spin-current exists. This is just the opposite situation of the traditional charge current without any spin. A pure spin current without an accompanying charge current, in the form of Fig. 1(a), has recently been experimentally demonstrated in semiconductor nanostructures with optical control. ${ }^{6}$

By Ampere's law, a charge current induces a magnetic field in the space surrounding it. In this paper, we ask and answer the following questions: can a pure steady-state spincurrent without charge current induce an electric field? If so, what are the properties of this induced electric field? The problem can be viewed in the following way. Associated with the electron spin $\boldsymbol{\sigma}$, there is a magnetic moment (MM) $g \mu_{B} \boldsymbol{\sigma}$, where $\mu_{B}$ is the Bohr magneton and $g$ is a constant. Therefore when there is a spin-current $I_{s}$, there is a corresponding MM current $I_{m}=g \mu_{B} I_{s}$. In the rest of the paper, we theoretically prove that a pure MM current with zero total MM can induce an electric field. It has been well known for decades that a single moving MM is equivalent to an electric dipole, ${ }^{7}$ it therefore follows that an infinite one-dimensional line of moving MM's not only has its associated magnetic field, it can also induce an electric field. ${ }^{8-10}$ Recently, Hirsch had given a formula for the electric field induced by a single moving magnetic dipole. ${ }^{8} \mathrm{He}$ also $\operatorname{argued}^{8}$ that this electric

field should survive in the case without a charge current and without a net magnetization. However, in order to unambiguously establish that the induced electric field is indeed due to spin current, not due to any other source such as the time varying magnetic field in the single moving MM case, in the present paper, we investigate the field induced by a steadystate spin-current in general situations where there is no charge, no charge current, and zero total spin. Importantly, we equally treat spin and charge as sources of electromagnetic field; and we systematically study the properties of the spin-current-induced field, by deriving several formula which play the role as the "Biot-Savart law" and "Ampere's law."

To start, we recall that a static classical MM m produces a magnetic field B. Consider a classical MM $\mathbf{m}$ due to a tiny charge-current ring, see Fig. 1(c). The charge-current is $I_{e c}$ and radius of the ring is $\delta$. The magnetic field $\mathbf{B}$ of this charge-current ring is easily obtained by the Biot-Savart law. Then, let $\delta \rightarrow 0^{+}$and $I_{e c} \rightarrow \infty$ but keep $\mathbf{m}=\pi \delta^{2} I_{e c} \hat{n}_{m}$ as a constant $\left(\hat{n}_{m}\right.$ is the unit vector of the MM), the magnetic field $\mathbf{B}$ due to $\mathrm{MM} \mathbf{m}$, at space point $\mathbf{R}$, can be written as

$$
\mathbf{B}=-\nabla \frac{\mu_{0} \mathbf{m} \cdot \mathbf{R}}{4 \pi R^{3}} .
$$
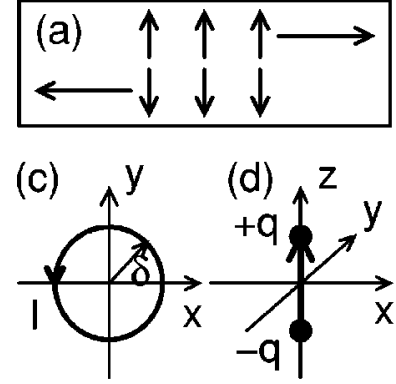

(e)

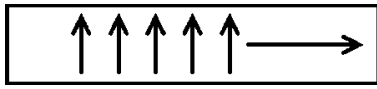

FIG. 1. Schematic plots for the spin current with zero charge current (a), the spin-polarized current (b), the MM of a small current ring (c), the two equivalent magnetic charges of the MM (d), and the straight infinite long MM line (e). (f) shows the electric (solid) and magnetic (dotted) line of force for the motive MM line. 
Another method for obtaining the same magnetic field is by using the mathematical construction of equivalent magnetic charge. ${ }^{7}$ In this method, we imagine the MM $\mathbf{m}$ being consisted of a positive and a negative magnetic charge $\pm q_{m c}$ situated very close to each other with a distance $\delta$ [see Fig. 1(d)]. When $\delta \rightarrow 0^{+}$and $q_{m c} \rightarrow \infty$, we hold $\mathbf{m}=q_{m c} \delta \hat{n}_{m}$ as a constant. Each magnetic charge $q_{m c}$ produces a magnetic field $\mu_{0} q_{m c} \mathbf{R} / 4 \pi R^{3}$. The field $\mathbf{B}$ induced by $\mathrm{MM} \mathbf{m}$ can then be obtained by adding contributions of the two magnetic charges $\pm q_{m c}$. Of course, we again obtain Eq. (1). Note that the language of magnetic charge is only a mathematical construction convenient for our derivations, ${ }^{7}$ and no magnetic monopole is hinted whatsoever.

After reviewing the magnetic field of a static MM, in the rest of the paper we consider MM's in motion. In the first example we consider the simplest case of a classical infinitely long one-dimensional (1D) lattice of chargeless MM, with the whole lattice moving with speed $\mathbf{v}$ [see Fig. 1(e)]. The induced field for this situation can be calculated exactly by a simple Lorentz transform, therefore providing a benchmark result for our more general results to be discussed later. Let $\rho_{m}$ be the linear density of MM for the lattice and we will use two methods to solve the electromagnetic field of the spin-current.

Method 1. We first solve the total magnetic field of a static 1D MM lattice by integrating Eq. (1) over the lattice. This is easy to do and we call the result $\mathbf{B}_{\text {static }}$ : $\mathbf{B}_{\text {static }}$ $=-\boldsymbol{\nabla}\left(\mu_{0} \rho_{m} \hat{n}_{m} \cdot \mathbf{R}_{\perp} / 2 \pi R_{\perp}^{2}\right.$, where $\mathbf{R}_{\perp}=\mathbf{R}-(\mathbf{R} \cdot \hat{l}) \hat{l}$. Here $\hat{l}$ is unit vector along the lattice. Then we make a relativistic transformation: the electromagnetic field of the moving MM lattice can be obtained straightforwardly by the Lorentz transform of $\mathbf{B}_{\text {static }}$. The results are

$$
\begin{gathered}
\mathbf{B}=\gamma \mathbf{B}_{\text {static }}, \\
\mathbf{E}=-\gamma \mathbf{v} \times \mathbf{B}_{\text {static }},
\end{gathered}
$$

where $\gamma=1 / \sqrt{1-\left(v^{2} / c^{2}\right)}$. Clearly, there has to be an induced electric field $\mathbf{E}$ and this field is related to $\mathbf{v} .{ }^{8}$ We note that although the results are unambiguously obtained, we have not identified the physical origin of the resulting electromagnetic field, i.e., this method does not tell us whether the field is induced by the MM or by the MM current. For this reason, we analyze the same problem again from a second method.

Method 2. Here we use the equivalent magnetic charge method discussed above. This means removing the current density of the ring at the equation $\boldsymbol{\nabla} \times \mathbf{B}=\mu_{0} \mathbf{J}_{e c}$ $+\mu_{0} \epsilon_{0}(\partial \mathbf{E} / \partial t)$, and adding the imaginary magnetic charge at the equation $\boldsymbol{\nabla} \cdot \mathbf{B}=0$, i.e., this equation changes to $\boldsymbol{\nabla} \cdot \mathbf{B}=\mu_{0} \rho_{m c}$, where $\rho_{m c}$ is the volume density of magnetic charge. We emphasize again that this practice is only a mathematical trick to solve our problem. When our MM moves, the original Maxwell equation in which the MM $\mathbf{m}$ is described by a tiny charge-current ring satisfies relativistic covariance. Clearly, Maxwell equations after the equivalent magnetic charge transformation must also satisfy relativistic covariance. This covariance can be achieved, as shown in standard textbook, ${ }^{7}$ by changing the Maxwell equation to $\boldsymbol{\nabla} \times \mathbf{E}=-\partial \mathbf{B} / \partial t$ to $\boldsymbol{\nabla} \times \mathbf{E}=-(\partial \mathbf{B} / \partial t)-\mu_{0} \mathbf{J}_{m c}$, where $\mathbf{J}_{m c}$ is the magnetic charge current. ${ }^{7}$ The last equation means that a moving magnetic charge can produce an electric field. The electric field $\mathbf{E}$ produced by a volume (linear) element of magnetic charge current, $\mathbf{J}_{m c} d V\left(I_{m c} d \mathbf{l}\right)$, is simply

$$
\mathbf{E}=-\frac{\mu_{0} \mathbf{J}_{\mathbf{m c}} d V \times \mathbf{R}}{4 \pi R^{3}}=-\frac{\mu_{0} I_{m c} d \mathbf{l} \times \mathbf{R}}{4 \pi R^{3}} .
$$

Now we are ready to solve the electromagnetic field of our 1D moving MM lattice because it is equivalent to two lines of positive/negative moving magnetic charges: they are easily obtained by integrating Eqs. (1) and (4), respectively. The same final results of Eqs. (2) and (3) are obtained. The present derivation allows us to conclude that the magnetic field $\mathbf{B}$ is induced by $\mathrm{MM}$ and the electric field $\mathbf{E}$ is induced by the MM current. Figure 1(f) shows electric-field lines and magnetic-field lines at the $y-z$ plane, here the infinitely long MM lattice is along the $x$ axis and $\hat{n}_{m}$ is along the $+z$ direction.

If there exists another infinitely long MM lattice with opposite MM direction $\left(-\hat{n}_{m}\right)$ and opposite moving direction [ $-\mathbf{v}$, shown in Fig. 1(a)], then the net MM is canceled exactly and only a net MM current exists. In this case, it is easy to confirm that the magnetic field $\mathbf{B}$ due to each lattice adds up to zero identically, while the electric field $\mathbf{E}$ reinforces each other so that the total electric field of the composite system is doubled. Hence we conclude that this finite electric field must originate from the MM current, and it cannot be due to any other effects.

In the example above, we have clearly shown that a classical 1D MM current can induce an electric field $\mathbf{E}$. In the following we investigate the question: can electron MM (i.e., spin) current induce an electric field? We also extend the above 1D model to general situation. Before proving this is indeed the case, we emphasize the fact that since a MM (or a spin) is itself a vector unlike charge which is a scalar, the MM current density cannot be described only by a single vector $\mathbf{J}_{m} d V$ (or $I_{m} d \mathbf{l}$ ). In order to completely describe a MM current density, ${ }^{11}$ we have to use a set of two vectors $\left(\hat{n}_{m}, \mathbf{J}_{m} d V\right)$ or $\left(\hat{n}_{m}, I_{m} d \mathbf{l}\right)$, in which $\mathbf{J}_{m}$ expresses the strength and direction of the flow of MM currents, while $\hat{n}_{m}$ expresses the polarization of the MM itself. This is different from the familiar charge current. Note that for two MM current, if only their $\mathbf{J}_{m}$ are the same and their $\hat{n}_{m}$ are different, they are two different MM currents and their induced electric fields are also different (see below).

In the following, we apply the equivalent magnetic charge method to deduce a general result beyond 1D for the quantum object of electron spin-current. Here, the spin or MM m of an electron at space point $\mathbf{r}$ is equivalent to a positive magnetic charge $m / \delta$ at $\mathbf{r}+(\delta / 2) \hat{n}_{m}$ and a negative magnetic charge $-m / \delta$ at $\mathbf{r}-(\delta / 2) \hat{n}_{m}$. The spin current $\left(\hat{n}_{m}, \mathbf{J}_{m} d V\right)$ at the space $\mathbf{r}$ is equivalent to two magnetic charge currents: one is $\left(\mathbf{J}_{m} / \delta\right) d V$ at $\mathbf{r}+(\delta / 2) \hat{n}_{m}$ and the other $-\left(\mathbf{J}_{m} / \delta\right) d V$ at $\mathbf{r}-(\delta / 2) \hat{n}_{m}$, where $\delta \rightarrow 0^{+}$. We make the very reasonable fundamental assumption that any electromagnetic field in- 
duced by moving electron spins, if exists, must satisfy relativistic covariance. From this assumption, the Maxwell equations for the magnetic charge and its current are

$$
\begin{gathered}
\boldsymbol{\nabla} \times \mathbf{E}=-\frac{\partial \mathbf{B}}{\partial t}-\mu_{0} \mathbf{J}_{m c}, \\
\boldsymbol{\nabla} \times \mathbf{B}=\mu_{0} \epsilon_{0} \frac{\partial \mathbf{E}}{\partial t}+\mu_{0} \mathbf{J}_{e c}, \\
\boldsymbol{\nabla} \cdot \mathbf{E}=\rho_{e c} / \epsilon_{0}, \\
\boldsymbol{\nabla} \cdot \mathbf{B}=\mu_{o} \rho_{m c},
\end{gathered}
$$

where $\rho_{m c}$ and $\rho_{e c}$ are the volume density of the magnetic and electric charge, $\mathbf{J}_{m c}$ and $\mathbf{J}_{e c}$ are their current density. In contrast, in the original Maxwell equation, the source of field are electric charge and its current: the field of a MM is calculated by turning this moment into an infinitesimal chargecurrent loop as we have done above. Here, we use the magnetic charge description and its associated current to express the spin of particles and the spin current. We emphasize three points.

(i) Equations (5)-(8) are more superior for our problem of calculating fields of electron spin-current because they do not require us to turn electron spins into little charge-current loops. No one knows how to do the latter, in fact, because the inner structure of an electron is not known. Hence, while the original Maxwell equations do not directly describe fields of electron spin and the spin current, Eqs. (5)-(8) can describe them and this description is very reasonable if we only investigate fields outside of an electron, e.g., for $R>10^{-5} \AA$.

(ii) Equations (5)-(8) do not represent an attempt of rewriting Maxwell equation. They are the Maxwell equation when we use equivalent magnetic charge and demanding relativistic covariance.

(iii) Note that although Eqs. (5)-(8) did appear in old literature, ${ }^{7}$ they were for describing totally different physics. For instance, ${ }^{7}$ if there exists a magnetic monopole, Maxwell equation will have the form of Eqs. (5)-(8). Since no monopole has ever been found, one recovers the standard form of Maxwell equation by setting $\mathbf{J}_{m c}$ and $\rho_{m c}$ to zero. ${ }^{7}$ In this work, we equally treat spin and charge as sources of electromagnetic field so that $\mathbf{J}_{m c}$ and $\rho_{m c}$ describe spin and spin current, without involving any magnetic monopole.

From Eqs. (5)-(8), the electric field induced by an infinitesimal element of magnetic charge current $\mathbf{J}_{m c} d V$ is obtained from Eq. (4). Then the total electric field $\mathbf{E}$ of the element of MM current $\left(\hat{n}_{m}, \mathbf{J}_{m} d V\right)$ can be calculated by adding the two contributions of the two magnetic charge currents: $\left(\mathbf{J}_{m} / \delta\right) d V$ at $(\delta / 2) \hat{n}_{m}$ and $-\left(\mathbf{J}_{m} / \delta\right) d V$ at $-(\delta / 2) \hat{n}_{m}$ $\left(\delta \rightarrow 0^{+}\right)$. We obtain ${ }^{12}$

$$
\mathbf{E}=\int \frac{\mu_{0}}{4 \pi} \mathbf{J}_{m} d V \times \frac{1}{R^{3}}\left[\hat{n}_{m}-\frac{3 \mathbf{R}\left(\mathbf{R} \cdot \hat{n}_{m}\right)}{R^{2}}\right] .
$$

Equation (9) clearly shows that the MM current $\left(\hat{n}_{m}, \mathbf{J}_{m} d V\right)$ (i.e., spin current $\left.\left(\boldsymbol{\sigma}, \mathbf{J}_{s} d V\right)=\left[\hat{n}_{m},\left(\mathbf{J}_{m} / g \mu_{B}\right) d V\right]\right)$ indeed can produce an electric field. This formula can be thought as the "Biot-Savart law" for spin-current-induced electric field. We emphasize that in the derivation of Eq. (9), the only assumption made was that the electromagnetic field of the moving spin satisfies relativistic covariance. As a check, applying Eq. (9) to the 1D lattice exactly solved above, it is straightforward to perform the integration and obtain Eq. (3).

Some further discussion of our results are in order. An electron has its charge and MM (spin): charge produces electric field, charge-current produces magnetic field, spin produces magnetic field, and we have just shown that a steady state spin-current produces an electric field.

(i) For the case of a spin current without charge current shown in Fig. 1(a), the total net charge is zero for our neutral system; the total charge-current is zero; and the total MM is also zero. The only nonzero quantity is the total spin-current (MM current). Our results predict even for this situation, an electric field is induced by the presence of spin current.

(ii) For the spin-polarized charge-current shown in Fig. 1(b), which have been extensively investigated recently, ${ }^{1,2}$ a charge current, total MM, and a spin current may all exist. In this case, the charge current and MM produce magnetic field, and the spin current produces electric field.

(iii) For a closed-loop circuit in which a steady state spin current flows, one can prove that the induced electric field $\mathbf{E}$ has the property $\oint_{c} \mathbf{E} \cdot d \mathbf{l}=0$, where $C$ is an arbitrary close contour not cutting the spin current. This is true even when the spin current threads the contour $C$ : very different from the Ampere's law of magnetic field induced by a charge current.

Figure 2 shows electric-field lines of a spin-current element $\left(\hat{n}_{m}, \mathbf{J}_{m} d V\right)$. The spin-current element is located at origin, $\mathbf{J}_{m}$ points to $+\mathbf{x}$ direction, and $\hat{n}_{m}$ is in the $\mathrm{x}-\mathrm{z}$ plane. The angle between $\mathbf{J}_{m}$ and $\hat{n}_{m}$ is $\theta$. Because the induced electric field $\mathbf{E}$ must be perpendicular to $\mathbf{J}_{m}$ (i.e., to $\mathbf{x}$ axis), we plot the field lines in the $y-z$ plane at $x=-1,0$, and +1 .

(i) For $\theta=\pi / 2, \hat{n}_{m} \perp \mathbf{J}_{m}$. At $x=0$, the field line configuration is similar (although not exactly the same) to that produced by an electric dipole in $-\mathbf{y}$ direction [Fig. 2(a)]. At $x= \pm 1$, the field lines have a mirror symmetry between upper and lower half $y-z$ plane [Fig. 2(b)].

(ii) For $\theta=0, \hat{n}_{m} \| \mathbf{J}_{m}$. At $x=0, \mathbf{E}=0$ for any $y$ and $z$. At $x= \pm 1$, the field lines are concentric circles [Fig. 2(c) and 2(d)]. The center of the circles is at $y=z=0$ where $\mathbf{E}=0$.

(iii) For $\theta=\pi / 3$, the fields are shown in Figs. 2(e) and 2(f) for $x= \pm 1$. In fact, this $\mathbf{E}$ can be decomposed into a summation of two terms corresponding to the fields of $\theta$ $=\pi / 2$ and $\theta=0$. At $x=0$, the field lines are similar to that shown in Fig. 2(a). It is worth mentioning that from Fig. 2, it is clearly shown that $\oint_{c} \mathbf{E} \cdot d \mathbf{l} \neq 0$. Note that this is not contradictory to characteristic (iii) of the last paragraph, because there the electric field is produced by a steady closed-loop spin-current.

So far we have demonstrated that a spin current can indeed induce an electric field. In the following, we investigate how a moving spin is affected by an external electromagnetic field. ${ }^{13}$ Consider a lab frame $\Sigma^{\prime}$ where there is an electromagnetic field $\left(\mathbf{E}^{\prime}, \mathbf{B}^{\prime}\right)$, and a static $\mathrm{MM} \mathbf{m}^{\prime}$. There is a 
(a): $\theta=\pi / 2, x=0$

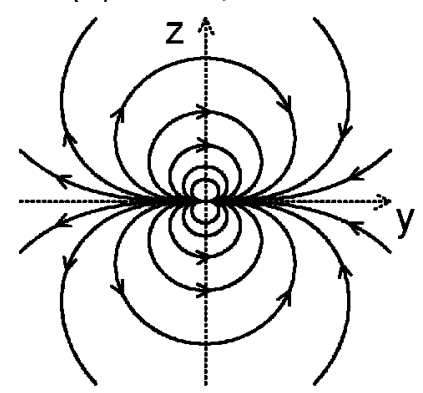

(c): $\theta=\mathbf{0}, \mathbf{x}=-\mathbf{1}$

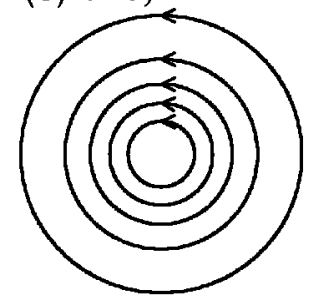

(e): $\theta=\pi / 3, x=-1$

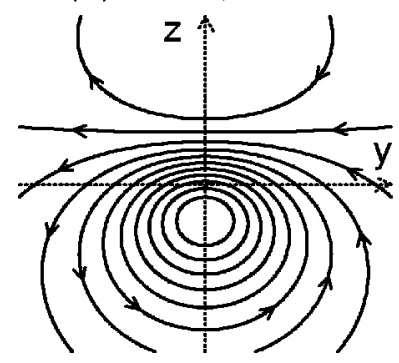

(b): $\theta=\pi / 2, x= \pm 1$

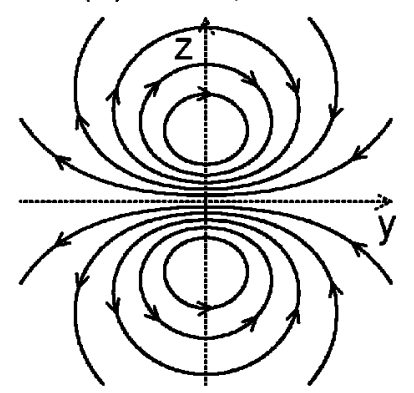

(d): $\theta=\mathbf{0}, \mathbf{x = 1}$
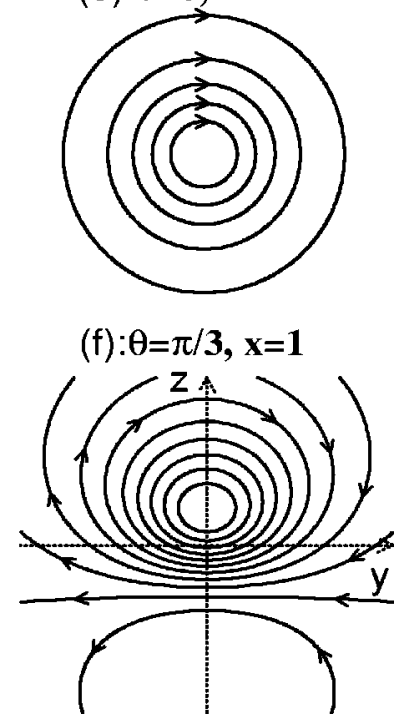

$(f): \theta=\pi / 3, x=1$

FIG. 2. Schematic plots of electric-field lines for a spin-current element with $\theta=\pi / 2, \pi / 3$, and 0 .

potential energy $-\mathbf{m}^{\prime} \cdot \mathbf{B}^{\prime}$ but $\mathbf{m}^{\prime}$ does not couple to $\mathbf{E}^{\prime}$. Using the language of magnetic charge discussed above, we can in effect consider that a force $q_{m c} \mathbf{B}^{\prime}$ is acting on the magnetic charge $q_{m c}$. Inside a new frame $\Sigma$ moving with speed $-\mathbf{v}$ respect to the lab frame $\Sigma^{\prime}$, the MM $\mathbf{m}$ (or the magnetic charge $q_{m c}$ ) move with speed $+\mathbf{v}$. A Lorentz transform of the four-momentum $p_{\mu}=(\mathbf{p}, i \mathrm{~W} / \mathrm{c})$ $=\left(p_{1}, p_{2}, p_{3}, i W / c\right)$ from frame $\Sigma^{\prime}$ to $\Sigma$ gives the force $\mathbf{F}$ on the moving magnetic charge $q_{m c}$ by the electromagnetic field $\quad \mathbf{E}, \mathbf{B}: \quad \mathbf{F}=d \mathbf{p} / d t=(d \mathbf{p} / d \tau)(d \tau / d t)=\left[d p_{1}^{\prime} / d \tau\right.$, $\left.\gamma^{-1}\left(d p_{2}^{\prime} / d \tau\right), \gamma^{-1}\left(d p_{3}^{\prime} / d \tau\right)\right]=q_{m c}\left(B_{1}^{\prime}, \gamma^{-1} B_{2}^{\prime}, \gamma^{-1} B_{3}^{\prime}\right)$ $=q_{m c}\left[\mathbf{B}-\left(\mathbf{v} / c^{2}\right) \times \mathbf{E}\right]$, where $\tau$ is the proper time, $t$ is the time, the quantity with a prime (without prime) is in frame $\Sigma^{\prime}(\Sigma)$, and the direction of $p_{1}$ is the same as velocity $\mathbf{v}$. Hence, a moving MM m (or spin $\sigma$ ) in an external electromagnetic field $\mathbf{E}, \mathbf{B}$ feels a torque: ${ }^{14} \mathbf{m} \times\left[\mathbf{B}-\left(\mathbf{v} / c^{2}\right) \times \mathbf{E}\right]$. Then the associated potential energy is:

$$
-\mathbf{m} \cdot\left(\mathbf{B}-\frac{\mathbf{v}}{c^{2}} \times \mathbf{E}\right)=-g \mu_{B} \boldsymbol{\sigma} \cdot\left(\mathbf{B}-\frac{\mathbf{v}}{c^{2}} \times \mathbf{E}\right) .
$$

Clearly, the term $-\mathbf{m} \cdot \mathbf{B}$ describes the action of magnetic field on $\mathbf{m}$ which is well known; and the term $\mathbf{m} \cdot\left[\left(\mathbf{v} / c^{2}\right)\right.$ $\times \mathbf{E}]$ expresses the action of electric field $\mathbf{E}$ on the moving MM.

So far we have found that a spin current can induce an electric field E; and conversely, an external electric field puts a moment of force on a spin current. The magnitudes of these effects can be estimated. Consider a spin current $\left(\hat{n}_{m}, \mathbf{J}_{m}\right)$ flowing in an infinitely long wire with cross section area of $2 \mathrm{~mm} \times 2 \mathrm{~mm}$. Let $\hat{n}_{m} \perp \mathbf{J}_{m}$, take electron density $10^{29} / \mathrm{m}^{3}$ and a drift velocity $10^{-2} \mathrm{~m} / \mathrm{s}$, then the spin-current-induced electric field is equivalent to that of a potential difference $\sim 12 \mu \mathrm{V}$ at distances $-1.1 \mathrm{~mm}$ and $1.1 \mathrm{~mm}$ on either side of the wire. This electric potential is indeed very small, but is definitely nonzero and should be measurable using present technologies.

We thank Professor D. Stairs for an illuminating discussion about electromagnetism. We gratefully acknowledge financial support from Natural Science and Engineering Research Council of Canada, le Fonds pour la Formation de Chercheurs et l'Aide à la Recherche de la Province du Québec, and NanoQuebec (Q.S., H.G,)n, a RGC grant from the SAR Government of Hong Kong under Grant No. HKU 7091/01P (J.W.), and NSFC under Grant No. 90303016(Q.S.)
${ }^{1}$ G.A. Prinz, Science 282, 1660 (1998).

${ }^{2}$ S.A. Wolf, D.D. Awschalom, R.A. Buhrman, J.M. Daughton, S. von Molnar, M.L. Roukes, A.Y. Chtchelkanova, and D.M. Treger, Science 294, 1488 (2001).

${ }^{3}$ A. Brataas, Y. Tserkovnyak, G.E.W. Bauer, and B. Halperin, Phys. Rev. B 66, 060404 (2002).

${ }^{4}$ J.E. Hirsch, Phys. Rev. Lett. 83, 1834 (1999).

${ }^{5}$ B. Wang, J. Wang, and H. Guo, Phys. Rev. B 67, 092408 (2003); Q.-F. Sun, H. Guo, and J. Wang, Phys. Rev. Lett. 90, 258301 (2003); W. Long, Q.-F. Sun, H. Guo, and J. Wang, Appl. Phys. Lett. 83, 1397 (2003).

${ }^{6}$ M.J. Stevens, A.L. Smirl, R.D.R. Bhat, A. Najmaie, J.E. Sipe, and H.M. van Driel, Phys. Rev. Lett. 90, 136603 (2003); J. Hübner, W.W. Rühle, M. Klude, D. Hommel, R.D.R. Bhat, J.E. Sipe, and
H.M. van Driel, ibid. 90, 216601 (2003).

${ }^{7}$ D. J. Griffiths, Introduction to Electrodynamics (Prentice-Hall, Englewood Cliffs, NJ, 1989).

${ }^{8}$ J.E. Hirsch, Phys. Rev. B 60, 14787 (1999).

${ }^{9}$ J.E. Hirsch, Phys. Rev. B 42, 4774 (1990).

${ }^{10}$ F. Meier and D. Loss, Phys Rev. Lett. 90, 167204 (2003); F. Schutz, M. Kollar, and P. Kopietz, ibid. 91, 017205 (2003).

${ }^{11}$ In a recent paper, Capelle et al. [K. Capelle, G. Vignale, and B.L. Gyorffy, Phys. Rev. Lett. 87, 206403 (2001)] have described spin currents by using a tensor product of the spin vector and the electron current. In fact, our description, a set of two vectors $\left(\hat{n}_{m}, \mathbf{J}_{m} d V\right)$, is equivalent to their tensor. For example, from $\left(\hat{n}_{m}, \mathbf{J}_{m} d V\right)$, one also may introduce the spin-current tensor as $\mathbf{K} d V=\hat{n}_{m} \otimes \mathbf{J}_{m} d V$. For a many-body system, if each electron 
spin direction $\hat{n}_{j}$ and/or its moving direction $\mathbf{J}_{j}$ are different from others, the spin current can also be described by $\mathbf{K} d V$ $=\sum_{j} \hat{n}_{j} \otimes \mathbf{J}_{j} d V$. For the tensor description method, the spin current $\mathbf{K} d V$ induced electric field can be obtained by using a similar method as presented in the text:

$$
\mathbf{E}=\sum_{i=x, y, z} \int \frac{\mu_{0}}{4 \pi} \mathbf{K}_{i} d V \times \frac{1}{R^{3}}\left[\hat{i}-\frac{3 \mathbf{R}(\mathbf{R} \cdot \hat{i})}{R^{2}}\right]
$$

where $\mathbf{K}_{i}=\left(K_{i x}, K_{i y}, K_{i z}\right)$.

${ }^{12}$ Note that Ref. 8 studied the electromagnetic field induced by a moving magnetic dipole $\mathbf{m}$. Reference 8 also mentioned a simi- lar result as Eq. (9). However, we emphasize that the induced electric field of spin current $\left(\hat{n}_{m}, \mathbf{J}_{m} d V\right)$ exists even when the total MM $\mathbf{m}$ is zero. This way, one unambiguously shows that the source of field was indeed the spin current.

${ }^{13}$ Note that the electric field acting on the moving spin [Eq. (10)] has been found before [C.-M. Ryu, Phys. Rev. Lett. 76, 968 (1996)]. Here we use the equivalent magnetic charge method to deduce it again.

${ }^{14}$ Notice when electromagnetic field $\mathbf{E}$ and $\mathbf{B}$ are nonuniform, the moving MM $\mathbf{m}$ feels a moment of force $\mathbf{m} \times\left[\mathbf{B}-\left(\mathbf{v} / c^{2}\right) \times \mathbf{E}\right]$. It is subjected to a net force $(\mathbf{m} \cdot \boldsymbol{\nabla})\left[\mathbf{B}-\left(\mathbf{v} / c^{2}\right) \times \mathbf{E}\right]$. 